\title{
Análisis de conectividad del paisaje para tres especies de mesodepredadores altoandinos como herramienta para su conservación ${ }^{1}$
}

\author{
Landscape connectivity analysis for three species of \\ high-andean predators as a tool for their conservation
}

Juan Sebastián Jiménez-Ramírez² (1) y Hugo Fernando López-Arévalo³ (1)

\section{RESUMEN}

Se evaluó la distribución potencial del cusumbo, zorro y oncilla en el norte de la Sabana de Bogotá, región de Colombia que presenta altas transformaciones por actividades antrópicas, encontrándose un área de 108749,8 ha disponibles para la distribución del cusumbo, 93899,64 ha disponibles para el zorro y 81014,15 ha disponibles para la oncilla. Además, se evaluó la conectividad funcional para las especies a partir de una distancia umbral determinada como el radio del área de acción reportados para cada especie y la conectividad a cinco veces el radio del área de acción, dando como resultado Índice Integral de Conectividad (IIC) bajos para las especies: 0,189 y 0,542 para el cusumbo, 0,212 y 0,417 para el zorro y de 0,185 y 0,361 para la oncilla. También se evaluó la conectividad de las especies en un escenario futuro, en el cual, los únicos fragmentos de hábitat disponible son los de la Reserva Forestal Protectora Productora Cuenca Alta del Río Bogotá, presentando una ausencia total en la conectividad para las tres especies a cualquier. Evidenciando la necesidad de implementar acciones para la preservación de los fragmentos de bosque existentes y aumentar su conectividad, con estrategias como las cercas vivas o reservas archipiélago.

Palabras clave: Mesodepredadores, fragmento, hábitat, Reserva Forestal Protectora Productora Cuenca Alta del Río Bogotá.

\begin{abstract}
The potential distribution of the cusumbo, fox and oncilla in the northern sector of the Sabana de Bogotá, region of Colombia that presents a high transformation due to anthropogenic activities, was evaluated, finding an area of 108749.88 ha available for the distribution of the cusumbo, 93899.64 ha available for the fox and 81014.15 ha available for the oncilla. In addition, functional connectivity for species was evaluated from a threshold distance determined as the radius of the area of action reported for each species and connectivity at a distance of five times the radius of the area of action, resulting in a Comprehensive Index of Low connectivity (IIC) for the three species: 0.189 and 0.542 for
\end{abstract}

Agradecemos a ONF Andina por suministrar material cartográfico, a la Universidad Nacional de Colombia y a la Convocatoria Nacional de Proyectos para el Fortalecimiento de la Investigación, Creación e Innovación de la Universidad Nacional de Colombia 2016-2018 por la financiación parcial y al grupo en Conservación y Manejo de Vida Silvestre de la Universidad Nacional de Colombia. Universidad Nacional de Colombia, Instituto de Ciencias Naturales. Correo electrónico: jsjimenezra@unal.edu.co Universidad Nacional de Colombia, Instituto de Ciencias Naturales. Correo electrónico: hflopeza@unal.edu.co 


\begin{abstract}
the cusumbo, 0.212 and 0.417 for the fox and 0.185 and 0.361 for the oncilla. The connectivity of the species was also evaluated in a future scenario, in which the only fragments of habitat available are those of the Protective Forest Reserve Producer Cuenca Alta del Río Bogotá, presenting a total absence of connectivity for the three species. Evidencing the need to implement actions for the preservation of existing forest fragments and increase their connectivity, with strategies such as live fences or archipelago reserves.
\end{abstract}

Keywords: Mesopredators, fragment, habitat, Reserva Forestal Protectora Productora Cuenca Alta del Río Bogotá.

La conectividad del paisaje es el grado en que el paisaje facilita o impide el movimiento entre parches de recursos, el intercambio genético y otros flujos ecológicos que son críticos para la viabilidad y supervivencia de las especies (Taylor et al., 1993; Crooks \& Sanjayan, 2006). Por lo tanto, la conectividad del paisaje es necesaria para sostener y mantener la estabilidad de los procesos ecológicos que se encuentran espacialmente relacionados entre sí, como la dispersión, el flujo genético entre poblaciones aisladas, la migración y a largo plazo la evolución de las especies (Taylor et al., 1993; Correa-Ayram et al., 2014), haciendo que sea esencial incluir la conectividad para la planificación de la conservación y el análisis del cambio del paisaje (Nikolakaki, 2004; Saura \& Pascual-Hortal, 2007a).

Los bosques montanos de la ecorregión del norte de los Andes son catalogados como uno de los sitios con mayor biodiversidad del mundo, desafortunadamente también se encuentran catalogados como unas de las zonas más amenazadas (Gómez Mora et al., 2005). La distribución de algunas poblaciones de plantas y animales han sufrido cambios drásticos debido a los cambios generados por el ser humano en los ecosistemas de todo el planeta, estos impactos han generado efectos importantes en la historia biológica del planeta, ocasionando grandes problemas ecológicos (Palma-Ordaz y Delgadillo-Rodríguez, 2014). La causa subyacente de prácticamente todas las disminuciones recientes y en curso de las especies de mamíferos es el crecimiento de las poblaciones humanas y los impactos asociados (Cardillo et al., 2004), siendo el cambio del uso del suelo para implementación de cultivos agrícolas el principal factor que influye en la sobrevivencia de las especies (Tilman et al., 2017).

Tres de las especies de mesodepredadores registradas en la zona de estudio son el cusumbo (Nasuella olivacea), el zorro (Cerdocyon thous) y la oncilla (Leopardus tigrinus) (Jiménez-Ramírez, 2019). El cusumbo y la oncilla son dos especies características de zonas de entre 1700 y $4100 \mathrm{~m}$, siendo más frecuentes sobre los 2000 m (Balaguera-Reina et al., 2009; Payán \& González-Maya, 2011). Estas dos especies presentan un gran vacío de información en aspectos ecológicos, desconociendo el estado de sus poblaciones, estando el cusumbo clasificado en la categoría de Casi Amenazado (NT) debido a que esta especie necesita de hábitats boscosos y gran parte de su rango de distribución se encuentra dentro de las áreas más pobladas y deforestadas de Colombia y Ecuador (González-Maya et al., 2016), por su parte, la oncilla se encuentra clasificada en la categoría de Vulnerable (VU) debido a que se estima que sus poblaciones han disminuido en un $31,7 \%$ en tres generaciones, además, es probable que las poblaciones presentes en centro américa sean otra especie diferente, al igual que ocurrió con las poblaciones presentes en el sur de Brasil ( $\mathrm{Pa}$ yan \& Oliveira, 2016), en cuando al zorro, es la especie de canido más común de las cinco presentes en el país (Suárez-Castro \& Ramírez-Chaves, 2015), registrándose en todo el territorio nacional a excepción de la región amazónica, entre los 0 y 3400 m (Solari et al., 2013; Suárez-Castro \& 
Ramírez-Chaves, 2015). Con el fin de discutir la conservación de estas tres especies, el objetivo del presente estudio es evaluar sus posibles zonas de distribución y la conectividad en el sector norte de la Sabana de Bogotá, Cundinamarca.

\section{Área de estudio}

El estudio se desarrolló en la zona norte de la Sabana de Bogotá, la cual abarca un total de 30 municipios. Encontrándose coberturas vegetales importantes para conservar la biodiversidad como lo son los bosques altoandinos y páramo. La zona de estudio alberga gran parte de la Reserva Forestal Protectora Productora Cuenca Alta del Río Bogotá, la cual es una reserva de Ley segunda, creada por la Junta Directiva del Instituto Nacional de Recursos Naturales y del Ambiente (INDERENA) con una extensión de 245147 ha (Ministerio de Ambiente y Desarrollo Sostenible, 1997; CAR et al., 2013). Sin embargo, en el momento de realizar dicha delimitación, existían en su interior áreas clasificadas como suelo urbano y zonas de expansión urbana, motivos por los cuales en el 2014 se realindera la reserva quedando con una extensión de 94161 ha (Ministerio de Ambiente y Desarrollo Sostenible, 1997). De los 28 fragmentos finales que conforma la reserva, en nuestro estudio por su ubicación incluimos 17 fragmentos (Figura $\mathrm{N}^{\circ} 1$ ).

Figura $\mathrm{N}^{\circ} 1$.

Sector norte de la Sabana de Bogotá Bosque, se consideran a las coberturas de bosque, arbustal y herbazal como hábitat
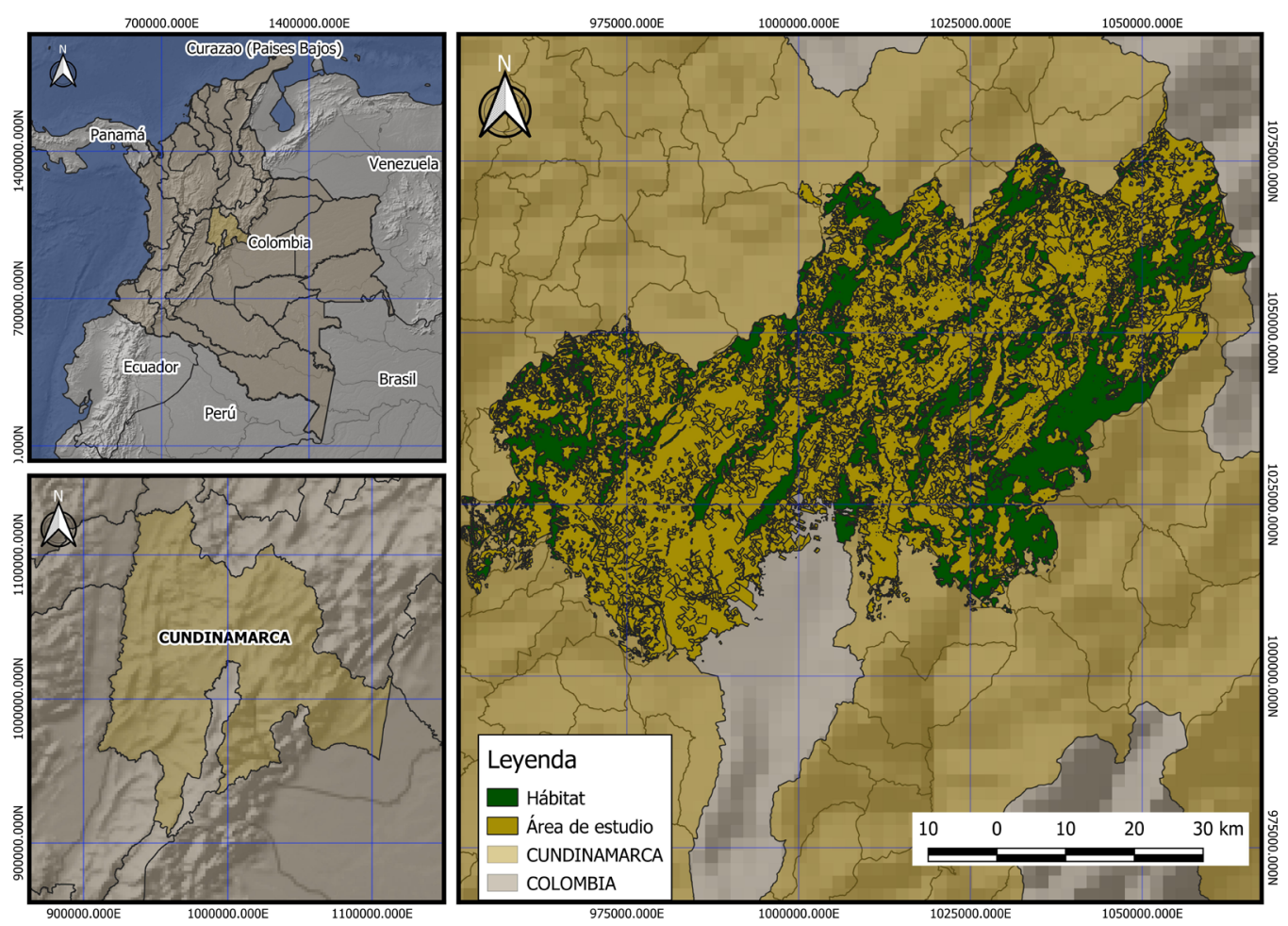

Fuente: Elaboración propia 


\section{Materiales y métodos}

\section{Clasificación de hábitat}

Clasificamos hábitat como las coberturas naturales en donde han sido reportadas al cusumbo, zorro y la oncilla (Nowell \& Jackson, 1996; Balaguera-Reina et al., 2009; Jiménez-Ramírez, 2019) estas coberturas fueron: bosques, arbustales, cultivos forestales y herbazales. Las coberturas que clasificamos como no hábitat fueron: pastos y potreros, cultivos transitorios y zonas urbanas. La clasificación se realizó por medio de cartografía de la zona a escala 1:25 000 suministrada por la entidad ONF-Andina, modificada empleando el Software de Sistema de Información Geográfico QGIS 3.6.3.

\section{Distribución potencial}

Los modelos de distribución realizados para estas especies incluyen al departamento de Cundinamarca con una alta probabilidad de distribución de éstas especies (Balaguera-Reina et al., 2009; Helgen et al., 2009; Payán \& González-Maya, 2011), no obstante, dada la alta transformación que sufre la región, asumimos que la distribución potencial de estas especies está directamente relacionada con las zonas en donde puedan encontrar refugio, alimentación y reproducción, así la delimitación a nivel local para las tres especies tuvo en cuenta el área de acción reportada en la literatura para cada especie, siendo para el cusumbo de 11,3 ha (Rodríguez-Bolaños et al., 2003), 280 ha para el zorro (Maffei \& Taber, 2003), para la oncilla, al ser una especie que no se ha evaluado su área de acción, se empleó el área de acción reportada para Leopardus guttulus de 1000 ha (Kasper et al., 2016). Además de plantear la distribución potencial actual para las tres especies de mesodepredadores, planteamos la distribución de estas especies en un escenario futuro, en donde los últimos fragmentos de hábitat disponibles dadas las dinámicas que presenta la zona estarían presentes dentro de la Reserva Forestal Protectora Productora Cuenca Alta del Río Bogotá. Por último, se identificaron registros de las tres especies en la zona de estudio, estos sitios de presencia de las especies se tomaron como la ocupación real.

\section{Evaluación de conectividad}

Para evaluar la conectividad se emplearon los fragmentos de distribución potencial de las tres especies, la evaluación de la conectividad se realizó calculando el Índice Integral de Conectividad (IIC) propuesto por Pascual-Hortal y Saura (2006).

$$
I I C=\frac{\sum_{i=1}^{n} \sum_{j=1}^{n} \frac{a_{i} \cdot a_{j}}{1+\mathrm{nl}_{i j}}}{A_{\mathrm{L}}^{2}}
$$

El Índice Integral de Conectividad (IIC) presenta valores de entre 0 y 1 , a medida que el valor se acerca a 1 significa que existe una mayor conectividad, en donde $\mathrm{n}$ corresponde al número total de nodos (en este caso los nodos corresponden a fragmentos de hábitat), ai y aj son atributos de los nodos $i$ y $j$, nlij es el número de enlaces en la ruta más corta entre fragmentos $i$ y $j$ y $A_{L}$ es el máximo atributo del paisaje, siendo en este caos tomado con el área total del paisaje, com- 
prendiendo zonas de hábitat y no hábitat (Pascual-Hortal \& Saura, 2006; Saura \& Pascual-Hortal, 2007a). El número de componentes se definió como el conjunto de fragmentos conectados a una distancia umbral definida, en este caso como el radio del área de acción de cada especie, y fue aumentando hasta llegar a un máximo de cinco veces la distancia inicial, además, se calculó la distancia mínima necesaria para que las especies perciba los fragmentos como un solo componente. Por último, se evaluó la importancia de conectividad acumulada (dIIC) de cada nodo (fragmento) en la zona de estudio.

\section{Resultados}

Para la zona de estudio se encontraron 17 localidades con registros del cusumbo, cinco localidades con registros del zorro y siete localidades con registros de la oncilla, los sitios en donde se encontraron los registros de las especies fueron considerados como nodos activos.

La zona de estudio presenta un área total de 455271,38 ha, de las cuales 109267,56 ha corresponden a hábitat, distribuidas en 354 fragmentos, presentando un tamaño promedio de 308,66 ha, el fragmento más pequeño presenta un área de 2,29 ha y el fragmento más grande un área de 28605,85 ha, la mayoría de los fragmentos se encuentran entre un área de 10 y 50 ha (Figura $\left.N^{\circ} 2 a\right)$. Si continúan las dinámicas de pérdida de las coberturas naturales por actividades antrópicas como la agricultura, ganadería entre otros, el escenario en el futuro sería muy diferente al que encontramos actualmente. En el escenario futuro que planteamos, la zona de estudio presentaría un área total de hábitat de 26721,04 ha, distribuidas en 108 fragmentos, presentando un área promedio de 247,42 ha, siendo el fragmento más pequeño de 0,14 ha y el más grande de 4198,83 ha, la mayoría de los fragmentos se encuentran entre los 10 y 50 ha (Figura $N^{\circ} 2 b$ ).

Figura $\mathrm{N}^{\circ} 2$.

Distribución de frecuencias de acuerdo con el área de fragmentos de bosques para la zona de estudio, fragmentos favorables para el cusumbo, fragmentos favorables para el zorro y fragmentos favorables para la oncilla. A. Escenario actual. B. Escenario futuro

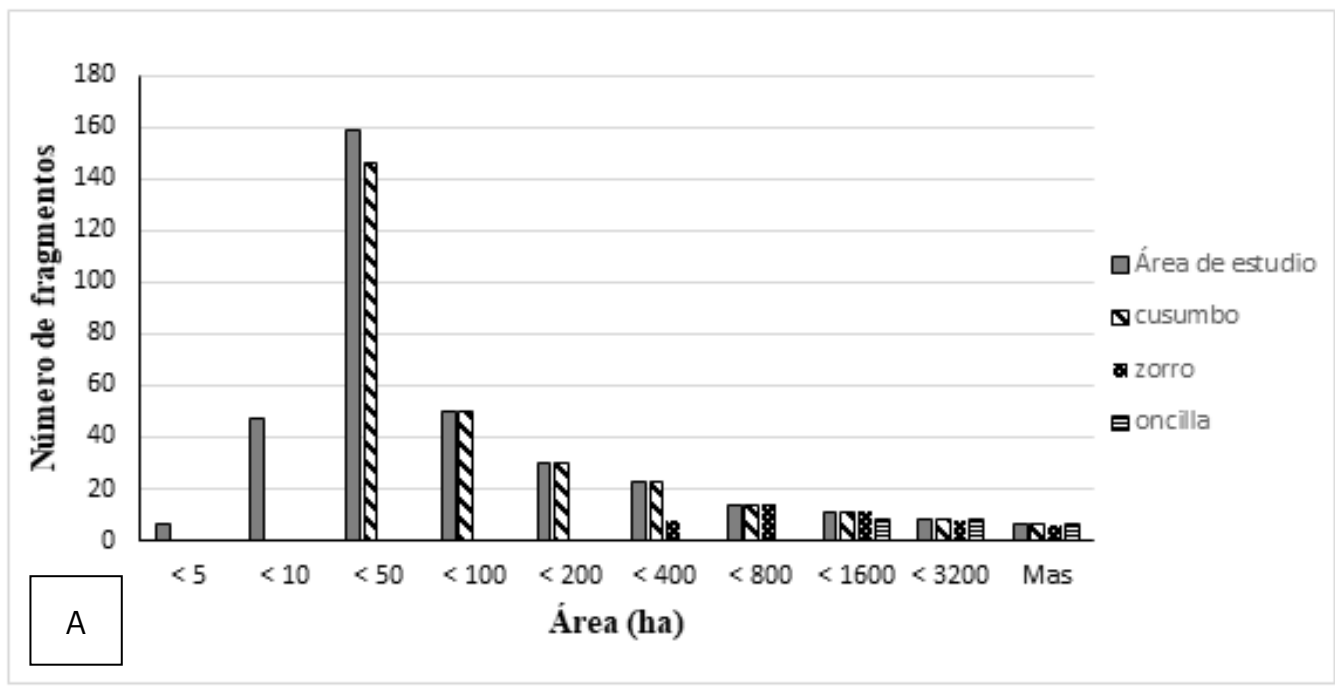




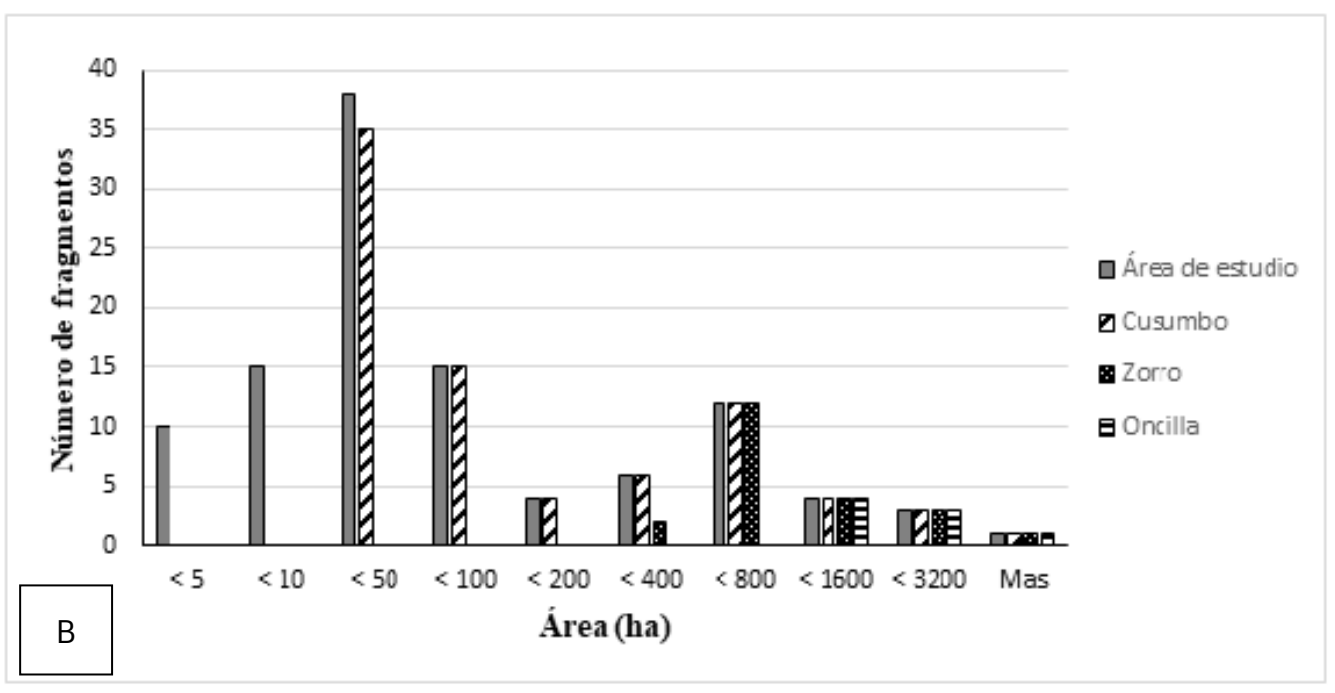

Fuente: Elaboración propia.

De los 354 fragmentos presentes en la zona de estudio, 288 cuentan con un área mínima para que el cusumbo pueda distribuirse, dando un área total de 108749,8 8 ha, un tamaño promedio de fragmento de 377,60 ha, siendo el área de fragmento más pequeño de 11,57 ha y el área más grande de 28605,85 ha (Cuadro $N^{\circ} 1$ y Figura ํ3). Para el zorro, 47 de los 354 fragmentos cuentan con el área mínima para que la especie esté presente, dando como resultado un área total de 93899,64 ha, con un tamaño promedio de 1997,86 ha, siendo el fragmento más pequeño de 300 ha y el fragmento más grande de 28605,85 ha (Cuadro $N^{\circ} 1$ y Figura $N^{\circ} 3$ ). Por último, para la oncilla la zona presenta 22 fragmentos con área mínima para la supervivencia de la especie, dando un área total de 81014,15 ha, un área promedio de 3682,46 ha, siendo el fragmento más pequeño de 1045,20 ha y el más grande de 28605,85 ha (Cuadro $N^{\circ} 1$ y Figura $N^{\circ} 3$ ).

\section{Cuadro $N^{\circ} 1$.}

Métricas de los fragmentos de hábitat en el área de estudio para las tres especies de mesodepredadores en el escenario actual y el escenario futuro

\begin{tabular}{|c|c|c|c|c|c|}
\hline Hábitat & Hábitat total & $\begin{array}{c}\mathbf{N}^{\circ} \\
\text { Fragmentos }\end{array}$ & $\begin{array}{c}\text { Fragmento } \\
\text { menor }\end{array}$ & $\begin{array}{c}\text { Fragmento } \\
\text { mayor }\end{array}$ & $\begin{array}{c}\text { Tamaño } \\
\text { promedio }\end{array}$ \\
\hline \multicolumn{6}{|c|}{ Escenario Actual } \\
\hline Área de estudio & 109267.56 & 354 & 2.29 & 28605.85 & 308.66 \\
\hline Cusumbo & 108749.88 & 288 & 11.57 & 28605.85 & 377.6 \\
\hline Zorro & 93899.64 & 47 & 300 & 28605.85 & 1997.86 \\
\hline Oncilla & 81014.15 & 22 & 1045.2 & 28605.85 & 3682046 \\
\hline \multicolumn{6}{|c|}{ Escenario futuro } \\
\hline Área de estudio & 26721.04 & 108 & 0.14 & 4198.83 & 247.42 \\
\hline Cusumbo & 26563.5 & 81 & 12.64 & 4198.83 & 332.04 \\
\hline Zorro & 23110.11 & 22 & 313.73 & 4198.83 & 1050.46 \\
\hline Oncilla & 15365.52 & 8 & 1172.97 & 4198.83 & 1920.69 \\
\hline
\end{tabular}

Fuente: Elaboración propia. 
Figura $\mathrm{N}^{\circ} 3$.

Distribución potencial de las tres especies de mesodepredadores en el sector norte de la sabana de Bogotá, en verde los fragmentos en donde se pueden distribuir las tres especies, en rojo los fragmentos en donde se pueden distribuir el zorro y el cusumbo y en amarillo los fragmentos en donde se puede distribuir el cusumbo

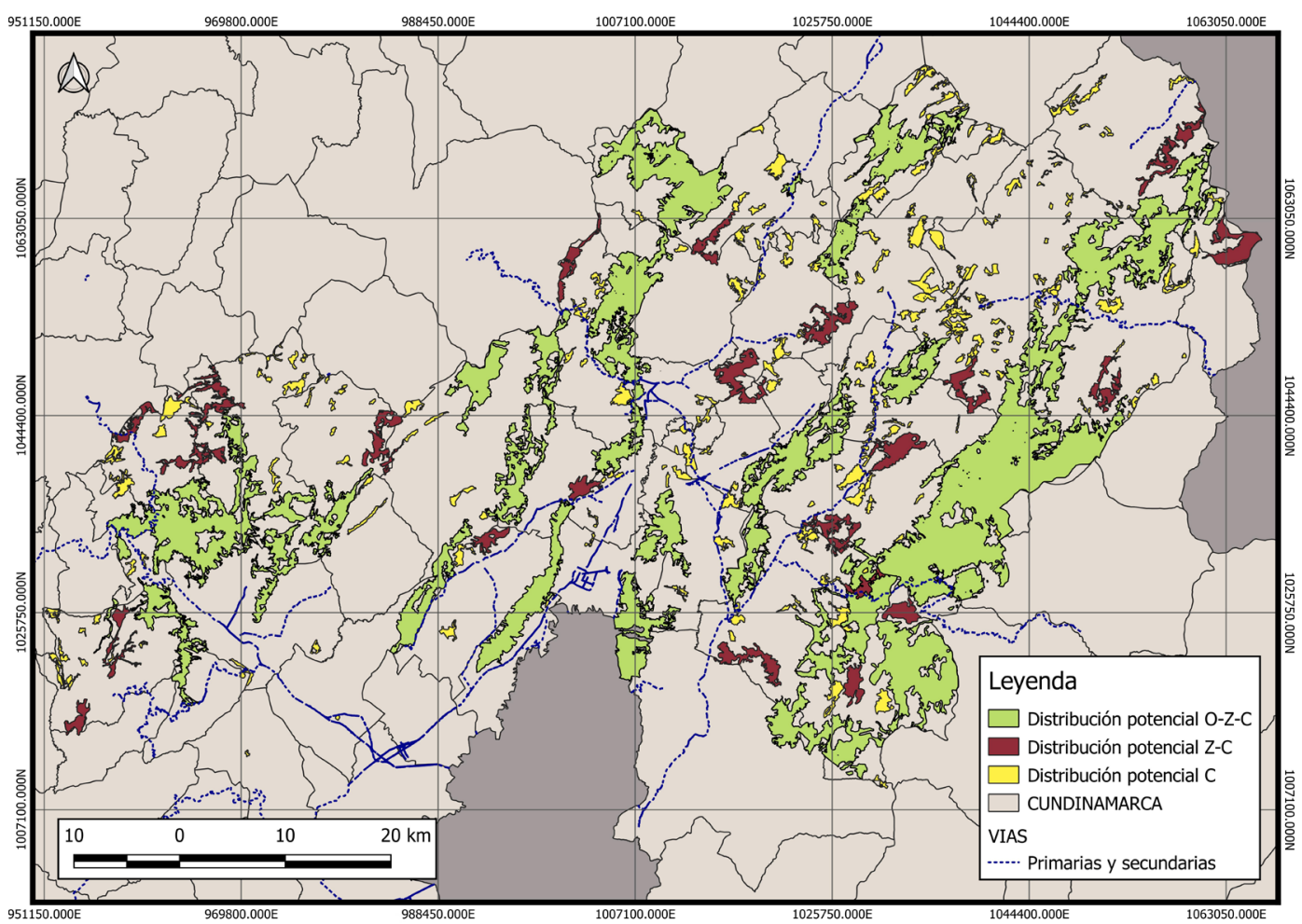

Fuente: Elaboración propia.

En el escenario futuro, de los 108 fragmentos presentes, 81 fragmentos presentan el área mínima para la supervivencia del cusumbo, con un área de 26563,50 ha, un tamaño promedio de 332,04 ha, siendo el fragmento más pequeño de 12,64 ha y el fragmento más grande de 4198,83 ha (Cuadro N¹ y Figura N4), para el zorro, 22 de los 108 fragmentos presentan área suficiente para su supervivencia, dando un área total de 23110,11 ha, un tamaño promedio de fragmento de 1050,46 ha, siendo el fragmento más pequeño de 313,73 ha y el más grande de 4198,83 ha (Cuadro $N^{\circ} 1$ y Figura $N^{\circ} 4$ ), por último, para la oncilla la zona de estudio presenta un área total de 15 365,52 ha, distribuidas en 8 fragmentos con un tamaño promedio de 1920,69 ha, siendo el fragmento más pequeño de 1172,97 ha y el más grande de 4198,83 ha (Cuadro №1 y Figura $\mathrm{N}^{\circ} 4$ ). 
Figura $\mathrm{N}^{\circ} 4$.

Distribución potencial de las tres especies en el escenario futuro en el sector norte de la Sabana de Bogotá, en verde los fragmentos en donde se pueden distribuir las tres especies, en rojo los fragmentos en donde se pueden distribuir el zorro y el cusumbo y en amarillo los fragmentos en donde se puede distribuir el cusumbo

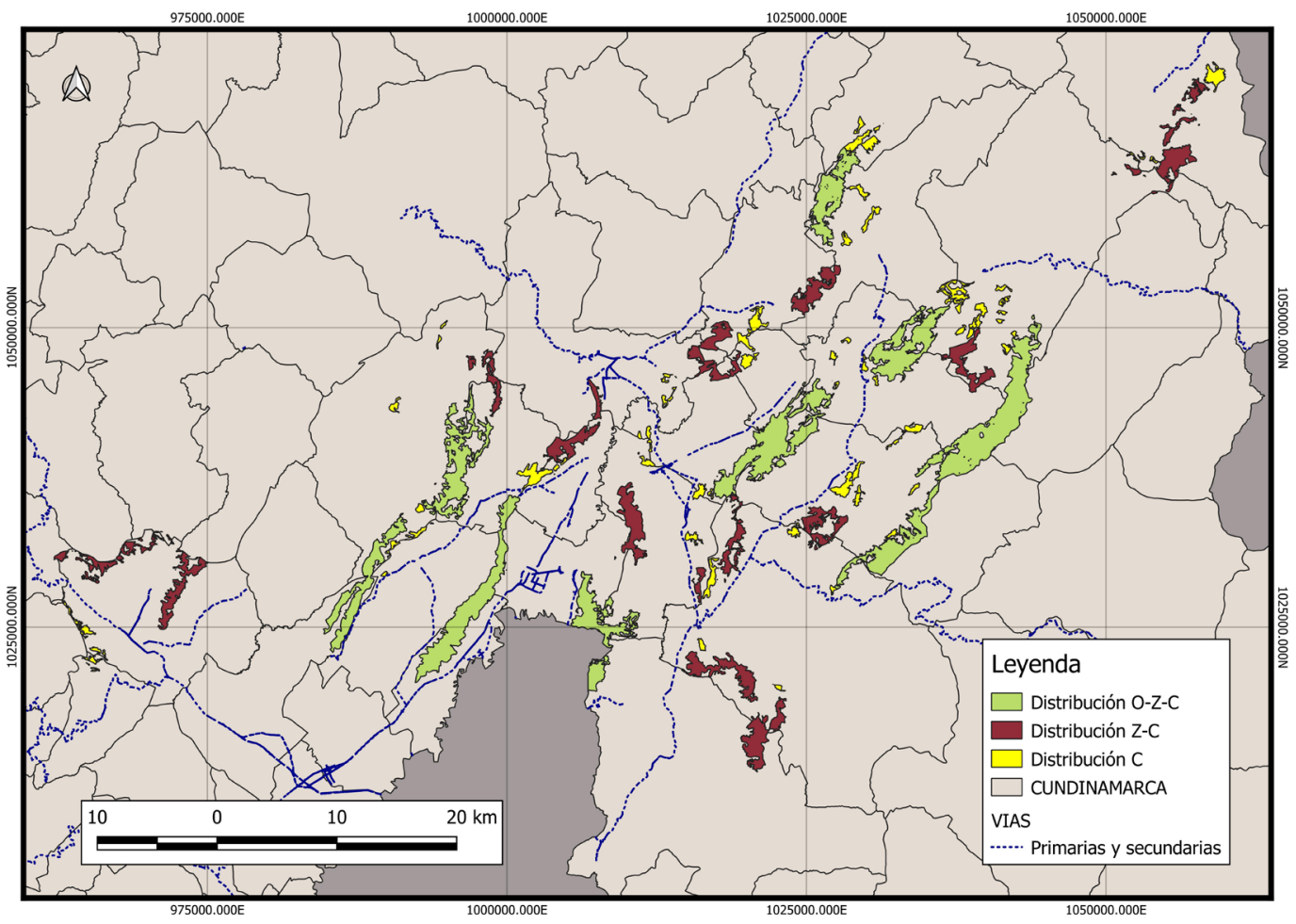

Fuente: Elaboración propia.

La identificación de número de componentes para el cusumbo muestra que a una distancia de $190 \mathrm{~m}$ los fragmentos forman un total de 161 componentes, mientras que a una distancia de desplazamiento de $950 \mathrm{~m}$ se forman un total de 39 componentes, siendo necesario que el cusumbo pueda desplazarse una distancia de $6170 \mathrm{~m}$ por la matriz de no hábitat para que pueda percibir todos los fragmentos como un solo componente (Figura $\mathrm{N}^{\circ} 5 \mathrm{a}$ ). En cuanto a la conectividad en el escenario futuro, a una distancia de $190 \mathrm{~m}$ los fragmentos forman un total de 48 componentes, a una distancia de desplazamiento para el cusumbo de $950 \mathrm{~m}$ se forman un total de 25 componentes, siendo necesario que ésta especie pueda desplazarse una distancia de $12690 \mathrm{~m}$ para que perciba todos los fragmentos como un solo componente (Figura $\left.N^{\circ} 5 a\right)$. Para el zorro, los fragmentos a una distancia de $944 \mathrm{~m}$ conforman un total de 19 componentes, a partir de los $4720 \mathrm{~m}$ los fragmentos de hábitat son percibidos por el zorro como un solo componente (Figura $\mathrm{N}^{\circ} 5 \mathrm{~b}$ ). En el escenario futuro, a una distancia de $944 \mathrm{~m}$ los fragmentos forman 18 componentes, a una distancia de desplazamiento de $4720 \mathrm{~m}$ se conforman un total de cinco componentes, siendo necesario que el zorro pueda desplazarse una distancia entre fragmentos de $14161 \mathrm{~m}$ para que los fragmentos de hábitat se comporten como un solo componente (Figura $N^{\circ} 5 b$ ). A una distancia umbral de $1784 \mathrm{~m}$ el hábitat de la oncilla está conformado por 11 componentes, a partir de los $8500 \mathrm{~m}$ el hábitat conforma un solo componente (Figura $\mathrm{N}^{\circ} \mathrm{5c}$ ), en cuanto al escenario futuro, a 
una distancia de desplazamiento de $1784 \mathrm{~m}$ el hábitat está conformado por ocho componentes, a una distancia de $8500 \mathrm{~m}$ se conforman un total de dos componentes, siendo necesario que esta especie pueda desplazarse una distancia entre la matriz de no hábitat de $11965 \mathrm{~m}$ para que el paisaje sea percibido por la especie como un solo componente (Figura $\mathrm{N}^{\circ} 5 \mathrm{c}$ ).

\section{Figura $\mathrm{N}^{\circ} 5$.}

Número de componentes en función de la distancia umbral. A) cusumbo, B) zorro y C) oncilla
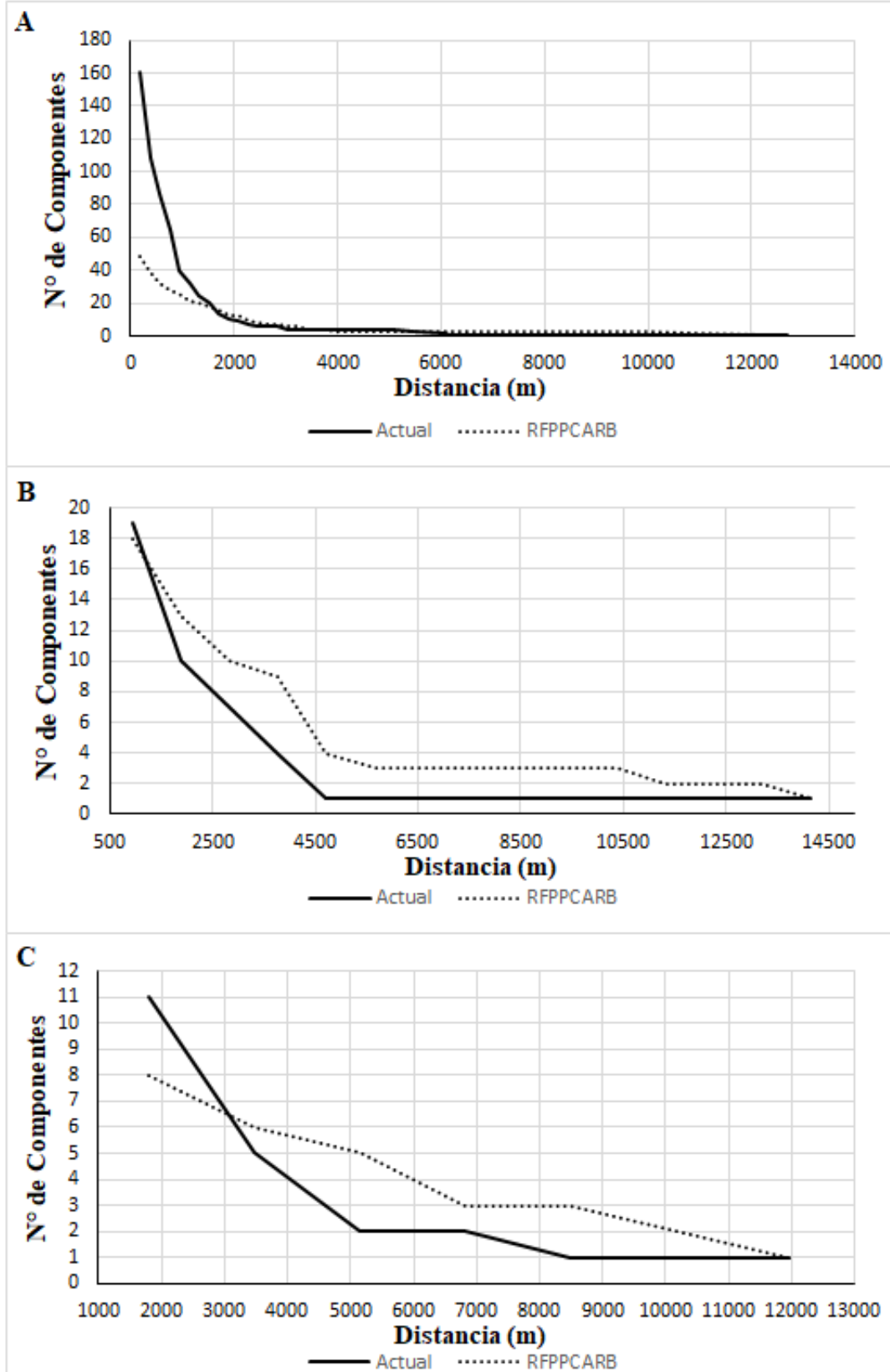

Fuente: Elaboración propia. 
El Índice Integral de Conectividad (IIC) es bajo para las tres especies en los dos escenarios, siendo aún más bajo en el escenario futuro, evidenciando que la zona de estudio presenta una alta separación entre los fragmentos de hábitat impidiendo la conectividad entre fragmentos para las tres especies, generándose fragmentos aislados de hábitat (Cuadro $\mathrm{N}^{\circ} 2$ ). Al analizar los valores de importancia de conectividad acumulada (dIIC) únicamente dos fragmentos presentan valores elevados $(12,28 \%$ y $79,28 \%)$ (Figura $\left.N^{\circ} 8\right)$.

\section{Cuadro $\mathrm{N}^{\circ} 2$.}

Resultados del Índice Integral de Conectividad (IIC) para las tres especies en tres distancias umbrales en los dos escenarios. Cusumbo: $\| \mathrm{CC} 1=190 \mathrm{~m}, \mathrm{IIC} 2=950 \mathrm{~m}$, IIC $3=6170 \mathrm{~m}$. Zorro: $\|\mathrm{C} 1=944 \mathrm{~m},\| \mathrm{C}$ $2=4720 \mathrm{~m}$. Oncilla: IIC $1=1784 \mathrm{~m}$, IIC $2=8920 \mathrm{~m}$. En el escenario futuro el IIC 3 se modificó para las tres especies. Cusumbo IIC $3=12690 \mathrm{~m}$. Zorro IIC $3=14161 \mathrm{~m}$. Oncilla IIC $3=11965 \mathrm{~m}$

\begin{tabular}{|c|c|c|c|c|c|c|}
\hline \multirow{2}{*}{ Especie } & \multicolumn{3}{|c|}{ Escenario actual } & \multicolumn{3}{c|}{ Escenario futuro } \\
\cline { 2 - 7 } & IIC 1 & IIC 2 & IIC 3 & IIC 1 & IIC 2 & IIC 3 \\
\hline Cusumbo & 0.190 & 0.257 & 0.542 & 0.0065 & 0.00725 & 0.0397 \\
\hline Zorro & 0.213 & 0.413 & - & 0.0062 & 0.01921 & 0.0317 \\
\hline Oncilla & 0.186 & 0.364 & - & 0.0052 & 0.00944 & 0.0157 \\
\hline
\end{tabular}

Fuente: Elaboración propia.

Figura $\mathrm{N}^{\circ} 6$.

Fragmentos de mayor importancia de conectividad acumulada (dIIC) en la zona de estudio

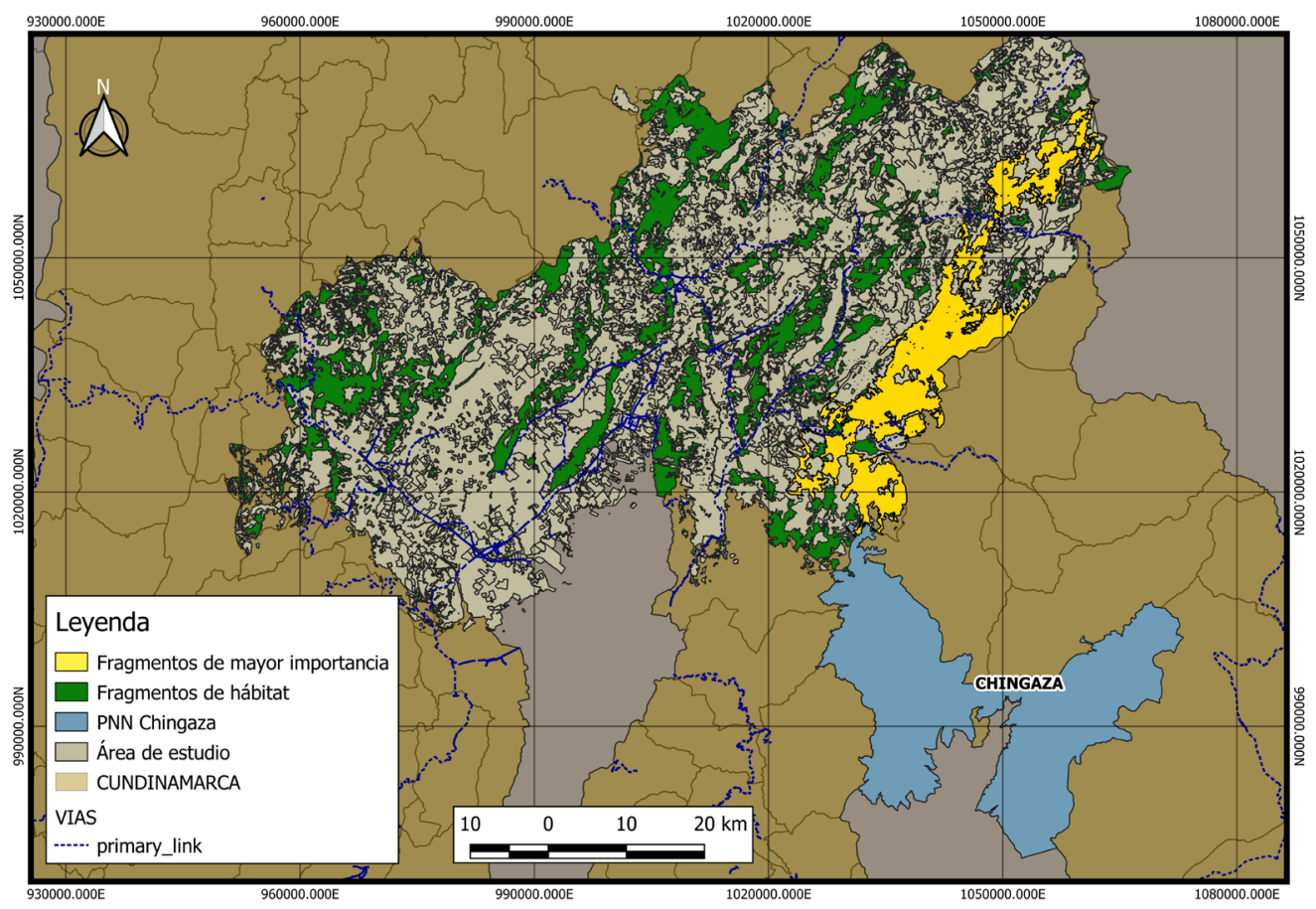

Fuente: Elaboración propia. 


\section{Discusión}

El grado en que el paisaje facilita o impide el movimiento entre fragmentos es un tema clave para la conservación de la biodiversidad y el mantenimiento de la integridad de los ecosistemas (Taylor et al., 1993; Saura \& Pascual-Hortal, 2007a). La baja conectividad encontrada explica que en el escenario inicial se generen una gran cantidad de componentes. Cuando la distancia entre fragmentos es mayor a la distancia en la que las especies pueden atravesar un paisaje transformado se reducen procesos ecológicos como la complementación y suplementación del paisaje, impidiendo que las especies puedan complementar o suplir los recursos necesarios con los presentes en otros fragmentos para su supervivencia (Dunning et al., 1992).

Al aumentar cinco veces la distancia de desplazamiento, la oncilla y el zorro podrían disponer de todo el hábitat al conformar un solo componente en el escenario actual, presentando de igual forma un aumento en los valores de IIC. Estas dos especie presentan cierto grado de tolerancia a la transformación, siendo la oncilla la especie del género Leopardus catalogada como la más tolerante a la transformación antrópica (Oliveira-Santos et al., 2012), de igual forma, la capacidad que presentan las especies de felinos de recorrer grandes distancias en busca de recursos alimenticios permitiría el paso de un fragmento a otro (Kasper et al., 2016), caso similar ocurre con varias especies de zorros, las cuales son tolerantes a la trasformación de sus hábitats por urbanización (Wandeler et al., 2003; Scott et al., 2014), siendo el zorro (C. thous) una especie que ha sido registrada dentro de ciudades de Colombia como Medellín-Antioquía ${ }^{4}$ y Florida Blanca-Santander ${ }^{5}$, demostrando la capacidad de adaptación que presenta esta especie a cambios en sus áreas naturales.

La conectividad de estos fragmentos es fundamental para la existencia de metapoblaciones, ya que puede permitirse el paso de individuos de un fragmento a otro, de esta forma mantener una alta diversidad genética, además de poder colonizar fragmentos de hábitat en diferentes temporadas, permitiendo una complementación y suplementación de los recursos, de igual for$\mathrm{ma}$, el proceso de fuente sumidero es fundamental en estas áreas fragmentadas, permitiendo el flujo de individuos desde fragmentos fuentes a fragmentos con condiciones no tan favorables consideradas (Dunning et al., 1992). López-Arévalo (2010) evaluó la conectividad de la zorra gris (Urocyon cinereoargenteus) en la cuenca alta del río La Antigua, Veracruz, encontrando que pese a la alta fragmentación y baja conectividad del bosque mesófilo, esta especie de zorro no se ve afectado debido a la configuración que presenta el estudio y a su capacidad de desplazamiento, estos resultados pueden ser comparados con los nuestros, en donde la oncilla y el zorro presentan una mayor conectividad gracias a sus capacidades de desplazamiento y a la tolerancia a zonas transformadas.

Por otro lado, un factor importante a tener en cuenta es la interacción que se puede presentar en las zonas de no hábitat y los bordes de los fragmentos entre estas especies de mesodepredadores nativas y los perros (Lacerda et al., 2009; López-Arévalo, 2010; Young et al., 2011; Rodríguez-León \& López-Arévalo, 2019). Algunos estudios muestran el impacto que ejercen los

Disponible en https://www.elcolombiano.com/medio-ambiente/por-que-hay-zorros-en-medellin-DC5895702 (10/08/2019)

Disponible en https://www.vanguardia.com/area-metropolitana/floridablanca/alerta-por-presencia-de-jauria-de-zorros-en-barrio-de-floridablanca-ODvl428006 (10/08/2019). 
perros sobre especies de canidos silvestres, modificando tiempo de forrajeo, abundancias relativas y sus distribuciones (p.e Vanak \& Gompper, 2009; Silva-Rodríguez et al., 2010; Paschoal et al., 2012; Zapata-Ríos \& Branch, 2016), de igual forma Payan y Oliveira (2016) reportan que una de las amenazas que presenta la oncilla en su área de distribución es la depredación por parte de los perros, además, Oliveira et al. (2013), Marinho (2015) y Sepúlveda et al. (2015) sugieren que otro efecto negativo ejercen los perros sobre la oncilla y el zorro es el contagio de enfermedades. No obstante, un factor que puede ayudar a minimizar el contacto entre los perros y las dos especies nativas son los patrones de actividad presentados por estas dos especies nativas, ya que tanto la oncilla como el zorro son especies principalmente nocturnas (Faria-Corrêa et al., 2009; Marinho et al., 2018), lo que le confiere una mayor facilidad para atravesar la matriz transformada entre un fragmento y otro, ya que de esta forma, reducen el contacto con los perros, ya que los patrones de actividad de esta especie doméstica han sido clasificados como diurnos (Sepúlveda et al., 2015; Jiménez-Ramírez, 2019; Rodríguez-León \& López-Arévalo, 2019).

En cuanto al cusumbo, encontramos que una gran parte de los fragmentos de hábitat se encuentran totalmente aislados, incluso al aumentar la distancia de desplazamiento cinco veces. Esta pérdida de conectividad es un tema crítico, para la persistencia de las poblaciones y es de vital importancia para generar estrategias de conservación (Dunning et al., 1992; Dunham \& Rieman, 1999). Lino et al. (2019) reportan que especies de mamíferos que se encuentran en situaciones de alta fragmentación del hábitat, su diversidad alélica se ve fuertemente reducida, siendo particularmente susceptible las especies que son dependientes de los bosques, como es el caso del cusumbo (Jiménez-Ramírez, 2019). Trabajos realizados por Arroyo-Rodriguez et al. (2005) con el mono aullador (Alouatta palliata mexicana) en los Tuxtlas, México y López-Arévalo (2010) con el puerco espín (Sphiggurus mexicanus) evidencian que por su baja capacidad de desplazamiento entre fragmentos, los procesos de fragmentación aumenta el aislamiento de las poblaciones, caso similar a lo que ocurre con el cusumbo en nuestra área de estudio, reduciendo su posibilidad de supervivencia ante la acelerada pérdida de coberturas naturales. A esta poca conectividad y el gran aislamiento que puede estar sufriendo el cusumbo se le adiciona la presencia de perros, los cuales ejercen un impacto negativo en las poblaciones de esta especie (Zapata-Ríos \& Branch, 2016; Jiménez-Ramírez, 2019).

Las especies que se encuentran bajo estos escenarios de alta fragmentación se ven obligados a moverse entre los fragmentos pequeños para poder cubrir sus requerimientos de hábitat (Zetterberg et al., 2010), por ende, el cusumbo tiene que arriesgarse a pasar por la matriz de no hábitat para llegar a otro fragmento, sin embargo, al ser una especie diurna (Rodríguez-Bolaños et al., 2003), el adentrase en esta matriz significa un gran peligro por la presencia de perros y otros factores antrópicos, ante esta amenaza, la actividad nocturna puede ser una estrategia para disminuir el peligro (Gaynor et al., 2018), siendo una especie catalogada como nocturna en la zona de estudio (Jiménez-Ramírez, 2019), de esta forma poder trasladarse de un fragmento a otro disminuyendo la probabilidad de muerte por depredación. De igual forma, en la zona de estudio se observa una reducción en el tamaño de grupo, siendo registrados únicamente individuos solitarios (Jiménez-Ramírez, 2019) siendo esta una especie altamente social, llegando a registrarse grupos de hasta 60 individuos en zonas conservadas (López-Arévalo et al., 1993; Sánchez \& Alvear, 2003), pese a que la reducción en el tamaño del grupo en una especie social es un impacto negativo importante, la disminución del tamaño de grupo puede favorecer un poco el traslado de un fragmento a otro, ya que estando solitarios son menos visibles para los depredadores. 
Otro factor importante que considerar en la conectividad de estas tres especies de mesodepredadores es la presencia de carreteras, desafortunadamente estas especies son reportadas por Delgado-V (2007; 2014) que sufre colisiones con vehículos en la vía el Escobero, ubicada en el municipio de Envigado, Antioquia, de igual forma, en la zona de estudio se registró a un cusumbo muerto en la vía y otro cruzando la vía a horas de la noche, evidenciando el riesgo que presentan estas especies en el momento de atravesar de un fragmento a otro, Fu et al. (2010) reportan que el impacto que generan las vías en la conectividad del paisaje en bosques de sureste de China, encontrado que las vías impactan negativamente la probabilidad de conectividad en un $32.78 \%$.

Si los procesos de transformación de las zonas urbanas continúan de la misma forma que van ocurriendo hasta el momento, el escenario futuro de las coberturas de hábitat se verá afectadas de una forma drástica, disminuyendo las zonas de distribución de las tres especies a unos cuantos fragmentos, y presentando valores de IIC cercanos a 0 , es decir que la conectividad para estas especies es casi inexistente. La reducción en el tamaño de los fragmentos da lugar a una progresiva pérdida de las especies, siendo una pérdida que se genera bajo un determinado orden (Patterson \& Atmar, 1986; Jackson \& Gaston, 2008), siendo las especies que presentan mayor área las primeras en desaparecer cuando el fragmento pierda ese tamaño umbral (Cardillo et al., 2004; Santos \& Tellería, 2006). Por lo tanto, en darse este escenario, las especies estarían aisladas y condenadas a desaparecer en el tiempo, caso similar es lo que ocurre con el lince ibérico, ya que las poblaciones remanentes de linces son pequeñas, se encuentran en aislamiento y presentan una baja variabilidad genética, convirtiéndolos en una especie que puede sufrir un descenso poblacional drástico con gran facilidad (Ministerio de Medio Ambiente y Medio Rural y Marino, 2008). Siendo fundamental implementar medidas en pro de conservar áreas prioritarias para la conservación de estas especies y que permitan generar conectividad entre estos fragmentos.

La cuantificación de la importancia individual de cada área de hábitat en términos de variación porcentual en el grado total de conectividad, permite un análisis adicional de apoyo a la toma de decisiones (Pascual-Hortal \& Saura, 2008), la zona de estudio solo presenta dos fragmentos con altos valores de importancia de conectividad acumulada (dIIC) (Figura $\mathrm{N}^{\circ} 6$ ), estos dos fragmentos son de gran relevancia para la conservación de las tres especies, ya que son los que presentan mayor conectividad, además, uno de estos dos fragmentos es el que presenta mayor área en la zona de estudio. De igual forma, este fragmento se encuentra en una ubicación de gran importancia, ya que la distancia entre este fragmento y la Parque Nacional Natural Chingaza es pequeña (Figura $\mathrm{N}^{\circ} 6$ ), siendo ésta una de las reservas más importantes de la región Andina, albergando a una gran diversidad de fauna, incluidas las especies de mesodepredadores registradas en nuestra zona de estudio (Vargas Ríos \& Pedraza, 2004; Jiménez-Ramírez, 2019).

Una estrategia que puede ser de gran utilidad por las características presentes en la zona de estudio es la creación de reservas archipiélago en la cual se incluyan estos dos fragmentos junto con zonas que se encuentran delimitadas dentro de la Reserva Forestal Productora Protectora Cuenca Alta del Río Bogotá, el objetivo de este tipo de reservas es cubrir lo más posible de la diversidad regional mediante complementariedad entre distintas áreas, permitiendo aumentar la conectividad, evitando el deterioro de la biodiversidad en conjuntos de paisajes que integran mosaicos regionales con distintos grados de transformación humana (Halffter, 2005). 
Además de la reserva archipiélago, aumentar la conectividad estructural de la zona de estudio por medio de corredores sería una acción fundamental para aumentar la conectividad de las especies entre los fragmentos. Una buena estrategia para aumentar la conectividad estructural es la creación de cercas vivas. Pese a que el origen de las cercas vivas tuvo lugar a la necesidad de resguardar al ganado en las zonas rurales, o a excluirlos de campos adyacentes (Martínez-Camilo et al., 2007), en términos de conservación las cercas vivas son consideradas como corredores dentro de los paisajes transformados, por lo tanto, su funcionalidad es característica de un corredor, generando un espacio adecuado para el movimiento o desplazamiento de organismos entre diferentes fragmentos de bosque (Chacón-León \& Harvey, 2007; Martínez-Camilo et al., 2007; De la Ossa-Lacayo, 2013), reduciendo los impactos que pueden sufrir las especies en matrices transformadas (García Quiroga \& Abad Soria, 2014).

\section{Conclusiones}

Debido a la alta transformación presente en la zona de estudio, el cusumbo, zorro y la oncilla presentan un área de distribución potencial de 108749,88 ha, 93899,64 ha y 81014,15 ha respectivamente, presentándose de igual forma una baja conectividad del paisaje para las tres especies a las menores distancias umbral de desplazamiento planteadas, sin embargo, al aumentar en cinco veces la distancia de desplazamiento, la oncilla y el zorro pueden percibir los fragmentos como un solo componente, caso contrario ocurre con el cusumbo, ya que dado su poca capacidad de desplazamiento, el paisaje está conformado por gran cantidad de componentes.

Al generar el posible escenario futuro de la zona de estudio, se presenta una reducción considerable en las distribuciones potenciales de las especies, dando como resultado un área de 26563,50 ha, 23110,11 ha y 15365,52 ha para el cusumbo, zorro y oncilla respectivamente, presentados una ausencia de conectividad entre los fragmentos de hábitat resultantes.

La generación de áreas de conservación que incluyan los fragmentos que presentan mayor conectividad junto con áreas dentro de la Reserva Forestal Protectora Productora Cuenca Alta del Río Bogotá es una alternativa importante para la preservación de las especies de mesodepredadores, siendo las reservas archipiélago una estrategia que resultaría benéfica para las tres especies de mesodepredadores estudiadas y otras especies de mamíferos presentes en el área de estudio, junto con la creación de cercas vivas que aumenten la conectividad entre los fragmentos presentes en la zona de estudio y de igual forma con el Parque Nacional Natural Chingaza.

\section{Referencias}

ARROYO-RODRIGUEZ, V., MANDUJANO, S., \& CUENDE-FANTON, C. Ocupación de parches de selva por monos aulladores Alouatta palliata mexicana en tres paisajes con diferente grado de fragmentación en los Tuxtlas, México. Univ. y Cienc., 2005, Vol. Especial, №2, p. 23-34.

BALAGUERA-REINA, S.A., CEPEDA, A., ZÁRRATE-CHARRY, D., \& GONZÁLEZ-MAYA, J.F. The state of knowledge of western mountain coati Nasuella olivacea in Colombia, and extent of occurrence 
in the Northern Andes. Small Carniv. Conserv. 2009, Vol. 41, p. 35-40. http://www.smallcarnivoreconservation.org/issue-41.html

Di BITETTI, M.S. Depredadores tope y cascadas tróficas en ambientes terrestres. Cienc. Hoy, 2008, Vol. 18, No108, p. 32-41.

CÁMARA DE COMERCIO DE BOGOTÁ. Caracterización económica y empresarial de diecinueve municipios de Cundinamarca. Bogotá, 2015.

CAR, CORPOGUAVIO, CONSERVACIÓN INTERNACIONAL \& ONF ANDINA. Componente de diagnóstico del plan de manejo de la cuenca alta del río Bogotá. Bogotá, 2015.

CAR, CORPOGUAVIO, MINISTERIO DE AMBIENTE Y DESARROLLO SOSTENIBLE Y INSTITUTO DE INVESTIGACIÓN DE RECURSOS BIOLÓGICOS ALEXANDER VON HUMBOLDT. Propuesta de realinderación y recategorización de la Reserva Forestal Protectora Productora la Cuanca alta del Río Bogotá declarada mediante el Artículo $2^{\circ}$ del Acuerdo 30 de 1976 aprobado por el Ministerio de Agricultura mediante la Resolución de 76 de 197. Bogotá, 2013.

CARDILLO, M., PURVIS, A., SECHREST, W., GITTLEMAN, J.L., BIELBY, J., \& MACE, G.M. Human population density and extinction risk in the world's carnivores. PLoS Biology, 2004, Vol. 2, N7, p. 909-914. https://doi.org/10.1371/journal.pbio.0020197

CHACÓN-LEÓN, M., \& HARVEY, C.A. Contribuciones de las cercas vivas a la estructura y conectividad de paisajes fragmentados en Río Frío, Costa Rica. En: HARVEY, C.A.; SÁEZ, J.C. (eds.). Evaluación y conservación de biodiversidad en paisajes fragmentados de Mesoamérica. Heredia: INBio, 2007, p. 226-248.

CORREA-AYRAM, C., MENDOZA, M.E., \& LÓPEZ-GRANADOS, E. Análisis del cambio en la conectividad del paisaje (1975-2008) de la cuenca del lago Cuitzeo, Michoacán, México. Rev. Geogr. Norte Gd., 2014, N59, p.7-23. https://dx.doi.org/10.4067/S0718-34022014000300002

COURTENAY, O., \& MAFFEI, L. Crab-eating fox Cerdocyon thous (Linnaeus, 1766). En: SILLERO-ZUBIRI, C.; HOFFMANN M.; MACDONALD, D.W. (eds.). Canids: Foxes, wolves, jackals and dogs. Status survey and conservation action plan. Gland, Siuza \& Cambridge, Reino Unido: IUCN/SSC Canid specialist group., 2004, pp. 32-38.

CROOKS, K.R. \& SANJAYAN, M. Connectivity conservation. New York: Cambridge University Press, 2006.

DE LA OSSA-LACAYO, A. Cercas vivas y su importancia ambiental en la conservación de avifauna nativa. Revista Colombiana de Ciencia Animal, 2013, Vol. 5, Nº1, p. 171-193. https://doi.org/10.24188/ recia.v5.n1.2013.483.

DELGADO-V, C.A. Muerte de mamíferos por vehículos en la vía del Escorbero, Envigado (Antioquia), Colombia. Actualidades Biológicas, 2007, Vol. 29, №87, p. 235-239. https://revistas.udea. edu.co/index.php/actbio/article/view/329342 
DELGADO-V, C.A. Adiciones al atropellamiento vehicular de mamíferos en la vía de el Escobero, Envigado (Antioquia), Colombia. Revista EIA, 2014, Vol. 11, N²2, p. 147-153. https://dx.doi. org/10.14508/reia.2014.11.22.147-153

DUNHAM, J.B., \& RIEMAN, B.E. Metapopulation structure of: influences of physical, biotic, and geometrical landscape characteristics. Ecological Applications, 1999, Vol. 9, N², p. 642-655. https://doi.org/10.1890/1051-0761(1999)009[0642:MSOBTI]2.0.CO;2

DUNNING, J.B., DANIELSON, B.J., \& PULLIAM, H.R. Ecological processes that affect populations in complex landscapes. OiKOS, 1992, Vol. 65, Nº1, p. 169-175. https://doi.org/10.2307/3544901

FARIA-CORREAA, M., BALBUENO, R.A., VIEIRA, E.M., \& DE FREITAS, T.R.O. Activity, habitat use, density, and reproductive biology of the crab-eating fox (Cerdocyon thous) and comparison with the pampas fox (Lycalopex gymnocercus) in a Restinga area in the southern Brazilian Atlantic Forest. Mammalian Biology, 2009, Vol. 74, p. 220-229. https://doi.org/10.1016/j.mambio.2008.12.005.

FU, W., LIU, S., DEGLORIA, S.D., DONG, S., \& BEAZLEY, R. Characterizing the "fragmentation-barrier" effect of road networks on landscape connectivity: a case study in Xishuangbanna, southwest China. Landscape and Urban Planning, 2010, Vol. 95, p. 122-129. https://dx.doi.org/10.1016/j.landurbplan.2009.12.009.

GARCÍA QUIROGA, F., \& ABAD SORIA, J. Los corredores ecológicos y su importancia ambiental: propuestas de actuación para fomentar la permeabilidad y conectividad aplicadas al entorno del río Cardeña (Ávila y Segovia). Observatorio Medioambiental, 2014, Vol. 17, p. 253-298. https://dx. doi.org/10.5209/rev_OBMD.2014.v17.47194.

GAYNOR, K.M., HOJNOWSKI, C.E., CARTER, N.H., \& BRASHARES, J.S. The influence of human disturbance on wildlife nocturnality. Science, 2018, Vol. 360, p. 1232-1235. DOI https://doi.org/10.1126/ science.aar7121.

GÓMEZ MORA, A.M., ANAYA, J.A., \& ÁLVAREZ DÁVILA, E. Análisis de fragmentación de los ecosistemas boscosos en una región de la cordillera central de los andes colombianos. Revi. Ing. Univ. Medellín, 2005, Vol. 4, no. 7, pp. 13-27. https://investigaciones-pure.udem.edu.co/es/publications/ an\%C3\%A1lisis-de-fragmentaci\%C3\%B3n-de-los-ecosistemas-boscosos-en-una-regi

GONZÁLEZ-MAYA, J.., REID, F. y HELGEN, K. Nasuella olivacea. IUCN Red List of Threat Species, 2016. https://dx.doi.org/10.2305/IUCN.UK.2016-1.RLTS.T72261737A45201571.en.

HALFFTER, G.Reservas archipiélago: un nuevo tipo de área protegida. En: HALFFTER, G.; GUEVARA, S.; MELO, A. (eds.). Hacia una cultura de conservación de la diversidad biológica. España, Zaragoza: Monografías Tercer Milenio, 2005, p. 281-286.

HELGEN, K.M., KAYS, R.W., HELGEN, L.E., TSUCHIYA-JEREP, M.T.N., PINTO, C.M., KOEPFLI, K.-P., EIZIRIK, E., \& MALDONADO, J.E. Taxonomic boundaries and geographic distributions revealed by an integrative systematic overview of the mountain coatis, Nasuella (Carnivora: Procyonidae). Small 
Carnivore Conservation, 2009, Vol. 41, p. 65-74. https://www.smallcarnivoreconservation.org/issue-41.html

JACKSON, S.F., \& GASTON, K.J. Land use change and the dependence of national priority species on protected areas. Global Change Biology, 2008, Vol. 14, p. 2132-2138. https://doi.org/10.1111/ j.1365-2486.2008.01628.x

JIMÉNEZ-ALVARADO, J.S., MORENO-DÍAZ, C., ALFONSO, A.F., GIORDANO, A.J., VELA-VARGAS, I.. M., GOMEZ-HOYOS, D., \& GONZALEZ-MAYA, J.F. Ciudades biodiversas: mamíferos medianos de la Reserva Forestal Protectora Bosque Oriental de Bogotá, D.C., Colombia. Mammal. Notes, 2017, Vol. 4, N¹, p. 37-41.

JIMÉNEZ-RAMÍREZ, J.S. Modelos de ocupación y distribución potencial de especies de mesodepredadores en el noroccidente de Cundinamarca, Colombia. Bogotá: Universidad Nacional de Colombia, 2019.

KASPER, C.B., SCHNEIDER, A., \& OLIVEIRA, T.G. Home range and density of three sympatric felids in the Southern Atlantic Forest, Brazil. Brazilian Journal of Biology, 2016, Vol. 76, №1, p. 228-232. https://doi.org/10.1590/1519-6984.19414

LACERDA, A.C.R., TOMAS, W.M., \& MARINHO-FILHO, J. Domestic dogs as an edge effect in the Brasília national park, Brazil: interactions with native mammals. Animal Conservation, 2009, Vol. 12, No5, p. 477-487. https://doi.org/10.1111/j.1469-1795.2009.00277.x

LIÉVANO-LATORRE, L.F., \& LÓPEZ-ARÉVALO, H.F. Comunidad de mamíferos no voladores en un área periurbana andina, Cundinamarca, Colombia. Acta Biol. Colomb., 2015, Vol. 20, №2, p. 193202. https://dx.doi.org/10.15446/abc.v20n2.43477

LINO, A., FONSECA, C., ROJAS, D., \& FISCHER, E. A meta-analysis of the effects of habitat loss and fragmentation on genetic diversity in mammals. Mammalian Biology, 2019, Vol. 94, p. 69-76. https://doi.org/10.1016/j.mambio.2018.09.006.

LÓPEZ-ARÉVALO, H.F. Efecto de la pérdida de conectividad del bosque mesófilo de montaña en la diversidad de mamíferos medianos en la cuenca alta del río La Antigua. Veracruz: Instituto de Ecología, 2010.

LÓPEZ-ARÉVALO, H.F., MONTENEGRO-DIAZ, O.L., \& CADENA, A. Ecología de los pequeños mamíferos de la Reserva Biológica Carpanta, en la cordillera oriental colombiana. Studies on Neotropical Fauna and Environment, 1993, Vol. 28, N4, p. 193-210. https://doi.org/10.1080/01650529309360904

MAFFEI, L., \& TABER, A.B. Área de acción, actividad y uso de hábitat del zorro patas negras, Cerdocyon Thous, en un bosque seco. Mastozoología Neotropical, 2003, Vol. 10, Nº1, p. 154-160. https:// www.redalyc.org/articulo.oa?id=45710113

MARINHO, P.H., BEZERRA, D., ANTONGIOVANNI, M., FONSECA, C.R., \& VENTICINQUE, E.M. Activity patterns of the threatened northern tiger cat Leopardus tigrinus and its potential prey in a Brazi- 
lian dry tropical forest. Mammalian Biology, 2018, Vol. 89, p. 30-36. https://dx.doi.org/10.1016/j. mambio.2017.12.004.

MARINHO, P.H. Gato-do-mato-pequeno (Leopardus tigrinus) na Caatinga: ocupação e padrão de atividade de um felídio ameaçado e pouco conhecido na floresta tropical seca do nordeste do Brasil. Natal: Universidade Federal do Rio Grande Norte, 2015.

MARTÍNEZ-CAMILO, R., MARTÍNEZ-MELÉNDEZ, N., \& PÉREZ FARRERA, M.Á. Las cercas vivas y su papel en la conservación de la biodiversidad en Chiapas. LACANDONIA, 2007, Vol. 1, №1, p. 117124. https://cuid.unicach.mx/revistas/index.php/lacandonia/article/view/102

MATEO, R.G., FELICÍSIMO, Á.M., \& MUÑOZ, J. Modelos de distribución de especies: una revisión sintética. Revista Chilena de Historia Natural, 2011, Vol. 84, p. 217-240. https://doi.org/10.4067/ s0716-078X2011000200008

MINISTERIO DE AMBIENTE Y DESARROLLO SOSTENIBLE. Resolución No. 0138. Bogotá, 1997.

MINISTERIO DE MEDIO AMBIENTE Y MEDIO RURAL Y MARINO. Estrategia para la conservación del lince ibérico (Lynx pardinus) en España. Madrid: Gráficas Arias Montano, 2008.

MORALES-MARTÍNEZ, D.M., SUAREZ-CASTRO, A.F., CÁRDENAS-CASTRO, C., \& FERNÁNDEZ-RODRÍGUEZ, C. Familia Procyonidae. En: SUÁREZ-CASTRO, A.F.; RAMÍREZ-CHAVES, H.E. (eds.). Los carnívoros terrestres y semiacuáticos continentales de Colombia. Guía de Campo. Bogotá: Editorial Universidad Nacional de Colombia, 2014, p. 136-164.

NIKOLAKAKI, P., 2004. A GIS site-selection process for habitat creation: estimating connectivity of habitat patches. Landsc. Urban Plan., vol. 68, pp. 77-94. https://doi.org/10.1016/S01692046(03)00167-1.

NOWELL, K., \& JACKSON, P. Wild cats. Status survey and conservation action plan. Ilinois: The Burlington Press. 1996.

OLIVEIRA-SANTOS, L.G.R., GRAIPEL, M.E., TORTATO, M.A., ZUCCO, C.A., CÁCERES, N.C., \& GOULART, F.V.B. Abundance changes and activity flexibility of the oncilla, Leopardus tigrinus (Carnivora: Felidae), appear to reflect avoidance of conflict. Zoologia, 2012, Vol. 29, №2, p. 115-120. https:// doi.org/10.1590/S1984-46702012000200003.

OliveIRA, T.G., TORTATO, M.A., de ALMEIDA, L.B., de CAMPOS, C.B., \& de BEISIEGEL, B.M. Avaliação do risco de extinção do Gato-do-mato Leopardus tigrinus (Schreber, 1775) no Brasil. Biodiversidade Brasileira, 2013, Vol. 31, p. 56-66.

PACHECO, V., CADENILLAS, R., SALAS, E., TELLO, C., \& ZEBALLOS, H. Diversidad y endemismo de los mamíferos del Perú. Revista Peruana de biología, 2009, Vol. 16, № 1, p. 5-32. https://doi. org/10.15381/rpb.v16i1.111. 
PACHECO, V., SALAS, E., CAIRAMPOMA, L., NOBLECILLA, M., QUINTANA, H., ORTIZ, F., PALERMO, P., \& LEDESMA, R. Contribución al conocimiento de la diversidad y conservación de los mamíferos en la cuenca del río Apurímac, Perú. Revista Peruana de Biologia, 2007, Vol. 14, №2, p. 169-180. https://doi.org/10.15381/rpb.v14i2.1722

PALMA-ORDAZ, S., \& DELGADILLO-RODRÍGUEZ, J. Distribución potencial de ocho especies exóticas de carácter invasor en el estado de Baja California, México. Bot. Sci., 2014, Vol. 92, №4, p. 587-597. https://doi.org/10.17129/botsci.135

PASCHOAL, A.M.O., MASSARA, R.L., SANTOS, J.L., \& CHIARELLO, A.G. Is the domestic dog becoming an abundant species in the Atlantic forest? A study case in southeastern Brazil. Mammalia, 2012, Vol. 76, N¹, p. 67-76. https://doi.org/10.1515/mammalia-2012-0501.

PASCUAL-HORTAL, L., \& SAURA, S. Comparison and development of new graph-based landscape connectivity indices: towards the priorization of habitat patches and corridors for conservation. Landscape Ecology, 2006, Vol. 21, p. 959-967. https://doi.org/10.1007/s10980-006-0013-z.

PASCUAL-HORTAL, L., \& SAURA, S. Integrating landscape connectivity in broad-scale forest planning through a new graph-based habitat availability methodology: Application to capercaillie ( $T e-$ trao urogallus) in Catalonia (NE Spain). European Journal of Forest Research, 2008, Vol. 127, p. 23-31. https://doi.org/10.1007/s10342-006-0165-z.

PATTERSON, B.D., \& ATMAR, W. Nested subsets and the structure of insular mammalian faunas and archipelagos. Biological Journal of the Linnean Society, 1986, Vol. 28, p. 65-82. https://doi. org/10.1111/j.1095-8312.1986.tb01749.x.

PAYÁN, E., \& GONZÁLEZ-MAYA, J.F. Distribución geográfica de la Oncilla (Leopardus tigrinus) en Colombia e implicaciones para su conservación. Rev. Latinoaméricana Conserv., 2011, Vol. 2, №1, p. 51-59.

PAYÁN, E. y OLIVEIRA, T. Leopardus tigrinus. IUCN Red List of Threat. Species, 2016. https://doi. org/10.2305/IUCN.UK.2016-2.RLTS.T54012637A50653881.en

RAMÍREZ-CHÁVES, H., \& PÉREZ, W.A. New record of crab-eating fox Cerdocyon thous in southwestern Colombia with comments on its distribution in Colombia and Ecuador. Canid biology and conservation, 2015, Vol. 18, No3, p. 6-9. https://www.canids.org/CBC/18/crab_eating_fox_in_Colombia.pdf.

RAMÍREZ-MEJÍA, A.F., \& SÁNCHEZ, F. Activity patterns and habitat use of mammals in an andean forest and a Eucalyptus reforestation in Colombia. Hystrix, Ital. J. Mammal., 2016, Vol. 27, №2, p. 1-7. Http://doi.org/10.4404/hystrix-27.2-11319.

RODRÍGUEZ-BOLAÑOS, A., SANCHEZ, P., \& CADENA, A. Patterns of activity and home range of mountain Coati Nasuella olivacea. Small Carnivore Conservation, 2003, Vol. 29, p. 16-19. https:// www.smallcarnivoreconservation.org/older-issues--volumes-26-32-.html 
RODRÍGUEZ-LEÓN, D.S., \& LÓPEZ-ARÉVALO, H.F. Variación de la abundancia relativa de perros en un gradiente de presencia humana en dos reservas (Tabio, Cundinamarca). Acta biologica Colombiana, 2019, Vol. 24, N², p. 379-390. https://doi.org/10.15446/abc.v24n2.70608

SÁNCHEZ-LONDOÑO, J.D. Diversidad y uso de hábitat de carnívoros (Carnivora) en un paisaje periurbano en la cordillera central de Colombia. Medellin: Universidad Nacional de Colombia, 2017.

SÁNCHEZ, F., GÓMEZ-VALENCIA, B., ÁLVAREZ, S.J., \& GÓMEZ-LAVERDE, M. Primeros datos sobre los hábitos alimentarios del tigrillo, Leopardus pardalis, en un bosque andino de Colombia. Rev. U.D.C.A Actual. Divulg. Científica, 2008, Vol. 11, № 2, p. 101-107. https://doi.org/10.31910/rudca.v11. $\mathrm{n} 2.2008 .627$

SÁNCHEZ, F.A., \& ALVEAR, M. Comentarios sobre el uso de hábitat, dieta, y conocimiento popular de los mamíferos en un bosque andino de Caldas, Colombia. Bol. Cient. Mus. Hist. Nat., 2003, Vol. 7, p. 121-144.

SANTOS, T., \& TELLERÍA, J L. Pérdida y fragmentación del hábitat: efecto sobre la conservación de las especies. Ecosistemas, 2006, Vol. 2, p. 3-12. https://doi.org/10.7818/re.2014.15-2.00.

SAURA, S., \& PASCUAL-HORTAL, L. A new habitat availability index to integrate connectivity in landscape conservation planning: comparison with existing indices and application to a case study. Landsc. Urban Plan., 2007A, Vol. 83, p. 91-103. https://doi.org/10.1016/j.landurbplan.2007.03.005.

SAURA, S., \& PASCUAL-HORTAL, L. Conefor Sensinode 2.2 User's manual: Software for quantifying the importance of habitat patches for landscape connectivity through graphs and habitat availability indices. España: Universidad de Lleida, 2007B.

SCOTT, D.M., BERG, M.J., TOLHURST, B.A., CHAUVENET, A.L.M., SMITH, G.C., NEAVES, K., LOCHHEAD, J., \& BAKER, P.J. Changes in the distribution of red foxes (Vulpes vulpes) in urban areas in Great Britain: findings and limitations of a media-driven nationwide survey. PLOS ONE, 2014, Vol. 9, Nº, p. 1-11. https://doi.org/10.1371/journal.pone.0099059.

SEPÚLVEDA, M., PELICAN, K., CROSS, P., EGUREN, A., \& SINGER, R. Fine-scale movements of rural free-ranging dogs in conservation areas in the temperate rainforest of the coastal range of southern Chile. Mammalian Biology, 2015, Vol. 80, p. 290-297. https://dx.doi.org/10.1016/j.mambio.2015.03.001

SILVA-RODRÍGUEZ, E.A., ORTEGA-SOLÍS, G.R., \& JIMÉNEZ, J.E. Conservation and ecological implications of the use of space by chilla foxes and free-ranging dogs in a human-dominated landscape in southern Chile. Austral Ecology, 2010, Vol. 35, p. 765-777. https://doi.org/10.1111/j.14429993.2009.02083.x

SOLARI, S., MUÑOZ-SABA, Y., RODRÍGUEZ-MAHECHA, J. V, DEFLER, T.R., RAMÍREZ-CHAVES, H.E., \& TRUJILLO, F. Riqueza, endemismo y conservación de los mamíferos de Colombia. Mastozool. Neotrop., 2013, Vol. 20, N², p. 301-365. https://mn.sarem.org.ar/issue/vol-20-no-2/ 
SUÁREZ-CASTRO, A.F., \& RAMÍREZ-CHAVES, H.E. Los carnívoros terrestres y semiacuáticos continentales de Colombia. Guía de Campo. Bogotá D.C: Editorial Universidad Nacional de Colombia, 2015.

TAYLOR, P.D., FAHRIG, L., HENEIN, K., \& MERRIAM, G. Connectivity is a vital element of landscape structure. Oikos, 1993, Vol. 68, №3, p. 571-573. https://doi.org/10.2307/3544927

TILMAN, D., CLARK, M., WILLIAMS, D.R., KIMMEL, K., POLASKY, S., \& PACKER, C. Future threats to biodiversity and pathways to their prevention. Nature, 2017, Vol. 456, p. 73-81. https:// doi,org/10.1038/nature22900.

VANAK, A.T., \& GOMPPER, M.E. Dietary niche separation between sympatric free-ranging domestic dogs and indian foxes in central India. Journal of Mammalogy, 2009, Vol. 90, N5, p. 1058-1065. https://doi.org/10.1644/09-MAMM-A-107.1

VARGAS RíOS, O., \& PEDRAZA, P. El Parque Nacional Natural Chingaza. Bogotá D.C: Gente Nueva Editorial, 2004.

WANDELER, P., FUNK, S.M., LARGIADĖR, C.R., GLOOR, S., \& BREITENMOSER, U. The city-fox phenomenon: genetic consequences of a recent colonization of urban habitat. Molecular Ecology, 2003, Vol. 12, p. 647-656. https://oi.org/10.1046/j.1365-294X.2003.01768.x

YOUNG, J.K., OLSON, K.A., READING, R.P., AMGALANBAATAR, S., \& BERGER, J. Is wildlife going to the dogs? Impacts of feral and free-roaming dogs on wildlife populations. BioScience, 2011, Vol. 61, N², p. 125-132. https://doi.org/10.1525/bio.2011.61.2.7

ZAPATA-RÍOS, G., \& BRANCH, L.C. Altered activity patterns and reduced abundance of native mammals in sites with feral dogs in the high Andes. Biological Conservation, 2016, Vol. 193, p. 9-16. http://dx.doi.org/10.1016/j.biocon.2015.10.016

ZETTERBERG, A., MÖRTBERG, U.M., \& BALFORS, B. Making graph theory operational for landscape ecological assessments, planning, and design. Landscape and Urban Planning, 2010, Vol. 95, p. 181-191. https://doi.org/10.1016/j.landurbplan.2010.01.002 
